\title{
Improving the Electrical Contact Property of Single-Walled Carbon Nanotube Arrays by Electrodeposition
}

\author{
Min Zhang*
}

(Received 10 August 2013; accepted 10 September 2013; published online 8 October 2013)

\begin{abstract}
A parallel method for the fabrication of metal contacts on single-walled carbon nanotube (SWNT) arrays was presented and the electrical contact property was evaluated by a SWNT-field effect transistor structure. Copper and gold contacts were fabricated on both semiconducting SWNTs and metallic SWNTs by using a maskless electrodeposition process. The SWNT array remained a p-type semiconductor after the electrodeposition. The contact resistance between SWNT array and microelectrodes was reduced more than $50 \%$ by the established copper contacts. The source-drain current of the carbon nanotube field-effect transistor (CNT-FET) structure can be further increased from $7.9 \mu \mathrm{A}$ to $9.2 \mu \mathrm{A}$ when the copper contacts were replaced by gold ones, which is probably due to the better contact property to SWNT of gold contacts with fine grain size.
\end{abstract}

Keywords: Carbon nanotube; Electrodeposition; Electrical contact

Citation: Min Zhang, "Improving the Electrical Contact Property of Single-Walled Carbon Nanotube Arrays by Electrodeposition", Nano-Micro Lett. 5(4), 242-246 (2013). http://dx.doi.org/10.5101/nml.v5i4. p242-246

\section{Introduction}

Since their discovery, single-walled carbon nanotubes (SWNTs) have been proposed as nanoscale building blocks for electronic, optoelectronic and sensing systems [1-4]. It is necessary to establish reliable electrical and/or mechanical contacts to the SWNTs for their integration in those systems. This has a space is needed, in the majority of the reported studies, by focused ion beam (FIB) or electron beam lithography (EBL). However, the sequential nature of FIB and EBL processes makes them time-consuming techniques and limits their application to the establishment of a small number of contacts. Other methods such as electron beam exposure [5] and adhesive [6] were also proposed, but they are still serial processes. Therefore, it is necessary to develop a parallel technique which can be used for the fabrication of large-scale SWNT-metal contact arrays for future applications. Zhang et al. reported a method to fabricate all-around contacts on SWMT by standard photolithography [7]. Contact improvement by the all-around contacts was found. However, potential contamination to SWNTs by the photoresist could lower the sensitivity of the SWNTs, especially when used as chemical or bio-sensors. Chen et al. proposed a method to bonding carbon nanotubes (CNTs) on metal electrodes by ultrasonic welding [8]. Long-term stable Ohmic contact between CNT and electrodes was achieved.

Recently, some efforts were made on fabricating metal contacts on CNTs by the electrodeposition method $[9,10]$. The problem lies in the fact that metals tend to grow not only on the microelectrdes contacting to CNTs, but also on the side wall of CNTs, which deteriorates the performance of the device. Actually, most reported work about electrodepositing metals on SWNTs result in metal nanoparticles or nanowires on the SWNT surface, especially for metallic single-walled

Advanced Manufacturing Division, Graduate School at Shenzhen, Tsinghua University, Shenzhen 518055, China

*Corresponding author. E-mail: zhang.min@sz.tsinghua.edu.cn 
carbon nanotubes (mSWNTs) and multi-walled carbon nanotubes $[11,12]$.

Previously, we have reported a parallel approach to fabricate copper contacts to single $\mathrm{ZnO}$ nanowire and SWNT using a selective electrodeposition process $[13,14]$. Effective contacts can be established by using a plating bath with low covering power at low electroplating potential. In the present work, both copper and gold contacts to SWNT arrays were fabricated using this maskless electrodeposition process, and their contact properties were evaluated using a field-effect transistor (FET) structure with SWNTs as the charge carrier channels. The effects of the microstructure of the deposited metal films and the deposition potential on the contact properties were also investigated.

\section{Experimental}

$\mathrm{Si}$ wafers covered with 500-nm thermally oxidized $\mathrm{SiO}_{2}$ were used as the substrates in this study. Paired comb-like $\mathrm{Cr} / \mathrm{Pt}$ electrode patterns were lithographically defined on the $\mathrm{SiO}_{2}$ layer. Well separated, long individual SWNTs with a controlled orientation were grown crossing the electrodes by catalytic chemical vapor decomposition (CVD) of ethanol, forming a FET structure [15].

Organic iodine Au ethanol solution (0.18 M KI, 0.12 $\left.\mathrm{M} \mathrm{I}_{2}, 0.02 \mathrm{M} \mathrm{Au}\right)$ and copper nitrate solution $(1.5 \mathrm{M}$ $\mathrm{Cu}\left(\mathrm{NO}_{3}\right)_{2}, 0.001 \mathrm{M} \mathrm{Cl}^{-}, 1.7 \mu \mathrm{M}$ PEG6000, $\left.\mathrm{PH} \approx 3\right)$ were used to deposit gold and copper contacts on the SWNT arrays, respectively. Both solutions work at room temperature. The deposition setup is depicted in Fig. 1. Two Pt contact electrodes were connected together to serve as the working electrode. A Pt wire with a diameter of $0.2 \mathrm{~mm}$ was used as a counter electrode during the fabrication of gold and copper contacts. To ensure the establishment of effective contacts, the deposition potentials were kept below $0.4 \mathrm{~V}$ and $0.2 \mathrm{~V}$

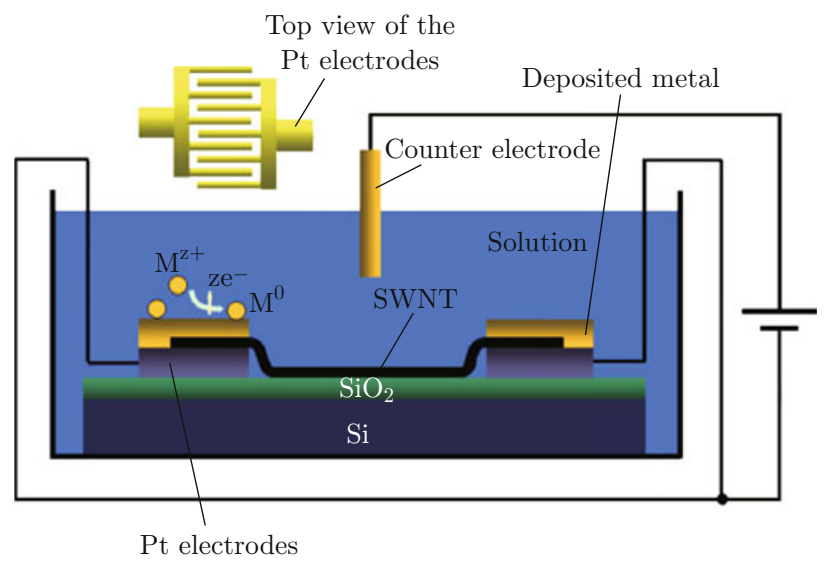

Fig. 1 Schematic illustration of the electrodeposition experimental set-up. for gold and copper deposition, respectively. Potentials of $0.5-0.8 \mathrm{~V}$ were also used for gold deposition in order to estimate the effect of deposition potential on the localization of deposited gold. After deposition, the samples were rinsed with a copious amount of ethanol and deionized water and dried in a nitrogen stream.

The post electrodeposited SWNT/electrode structures were analyzed using both field emission scanning electron microscope (FE-SEM) and atomic force microscopy (AFM). FE-SEM images were taken using a Sirion200 FE-SEM instrument. AFM images were acquired in tapping mode using an NT-MDT AFM instrument.

To evaluate the properties of electrodeposited contacts, a picoammeter (Keithley 6487) was used to measure the electrical properties of the SWNT-FET structures before and after metal deposition.

\section{Results and discussion}

Figure 2(a) shows the fabricated FET structure with SWNT array lying on Pt electrodes. The SWNT array is composed of individual SWNTs with length of tens of micrometers. Generally, in the SWNTs grown by CVD method, one third SWNTs are metallic, while the rest are semiconducting, depending on their diameter and chiral angle [16]. This is proved by the measurement of electrical properties of the SWNT-FET structure before electrodeposition. Figure 3(a) shows the source-drain
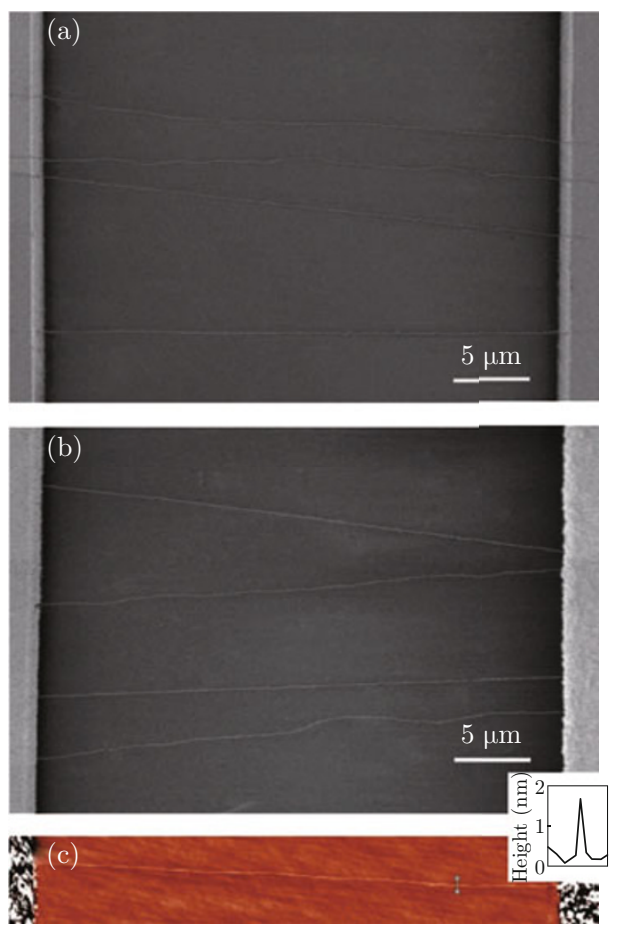

Fig. 2 SEM images of the SWNT array on Pt microelectrodes before (a) and after (b) gold electrodeposition. (c) AFM image of the SWNT after gold electrodeposition. 

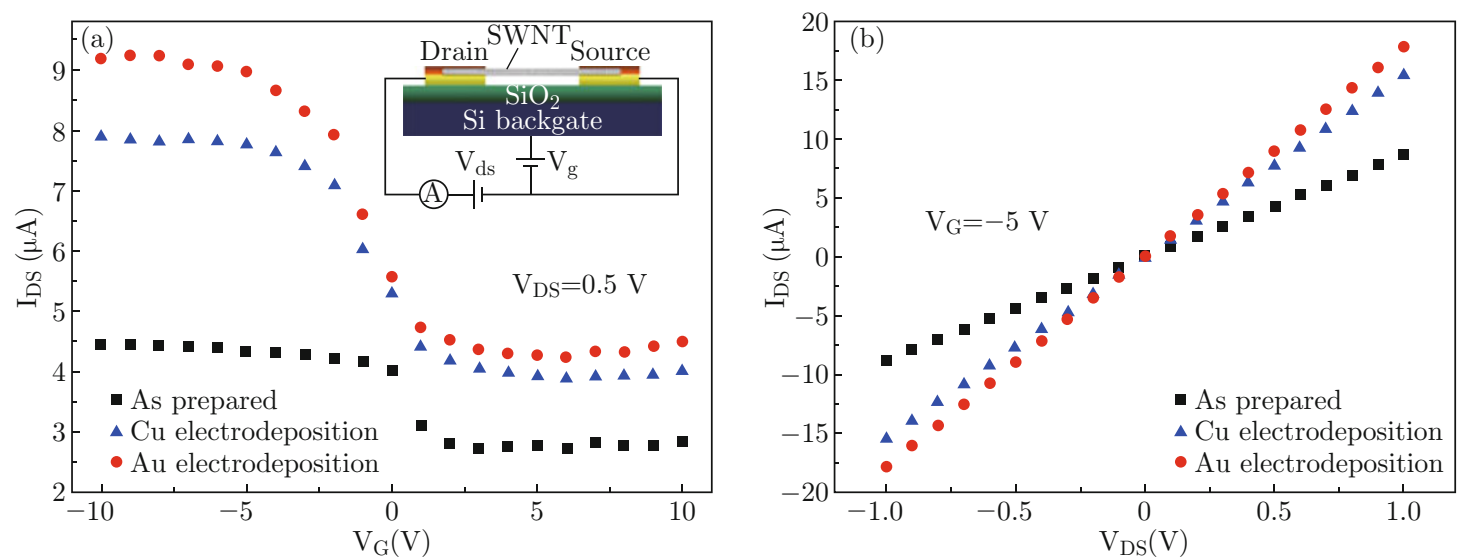

Fig. 3 (a) Charge transfer measurement of the SWNT-FET structure before and after electrodeposition of copper (at a deposition potential of $0.2 \mathrm{~V}$ ) and gold $(0.4 \mathrm{~V})$. The inset is the illustration of the wiring scheme for the measurement; (b) $I_{D S}$ as a function of $V_{D S}$ at $V_{G}=-5 \mathrm{~V}$.

current $I_{D S}$ versus gate bias $V_{G}$ for the SWNT array using a p-doped Si substrate as backgate. The decrease of $I_{D S}$ with increasing $V_{G}$ is consistent with the presence of semiconducting single-walled carbon nanotubes (sSWNTs), which are usually found to be 'p-type' doped [2]. The large source-drain leakage current (more than $2.7 \mu \mathrm{A}$ at $V_{D S}=0.5 \mathrm{~V}$ ) indicates the existence of $\mathrm{mSWNTs}$ whose resistivity is almost independent with gate bias.

After electrodeposition at a potential of $0.4 \mathrm{~V}$ in $\mathrm{Au}$ plating solution, the SWNTs were embedded in gold only on the prepatterned electrodes without the need of a mask to insulate the SWNTs between the electrodes, as shown in Fig. 2(b). The cleanliness of the CNT surface was further examined by AFM. Since the CNT surfaces have low coordination and interfacial energy, the nucleation of atoms on them tends to form atom clusters rather than a continuous monolayer [17]. As shown in Fig. 2(c), no particles were observed along the whole SWNTs, and the diameters of the post-deposited SWNTs were measured to be $1.2-1.8 \mathrm{~nm}$ which are the typical value of individual SWNTs in our samples [18]. Therefore, we consider the surface of SWNTs between the electrodes, including both sSWNTs and mSWNTs, is clean of gold. Copper contacts can also be realized on the SWNT array by using copper nitrate solution and controlling the deposition potential below $0.2 \mathrm{~V}$.

The charge transport measurements of the carbon nanotube field-effect transistor (CNT-FET) after electrodeposition were also shown in Fig. 3(a). After metal deposition, the SWNT array remained a p-type semiconductor. The on-current of the FET, which is $I_{D S}$ at negative $V_{G}$, increased from $4.5 \mu \mathrm{A}$ to $7.9 \mu \mathrm{A}$ at $V_{G}=$ $-10 \mathrm{~V}$ when using copper contacts. As gold contacts were applied, the on-current can be further improved to $9.2 \mu \mathrm{A}$, indicating the overall electrical resistance of the devices was reduced by the metal electrodeposition process. Figure 3(b) shows that the contact resistance of the device was reduced from $118 \mathrm{k} \Omega$ to $64 \mathrm{k} \Omega$ and 56 $\mathrm{k} \Omega$ by copper and gold deposition, respectively.

The better performance of gold contacts can be explained by the different microstructures of the electrodeposited gold and copper films. As shown in Fig. 4, the grain size of the deposited copper film is much larger than that of the gold film. Since the diameter of the SWNT is only 1-2 nm, metal film with a fine grain size could show better contact performance than that with coarse microstructures. The effect of microstructure of the deposited layer on their contact properties will be our future work.

The electroplating potential plays a very important role in controlling the localization of the electrode
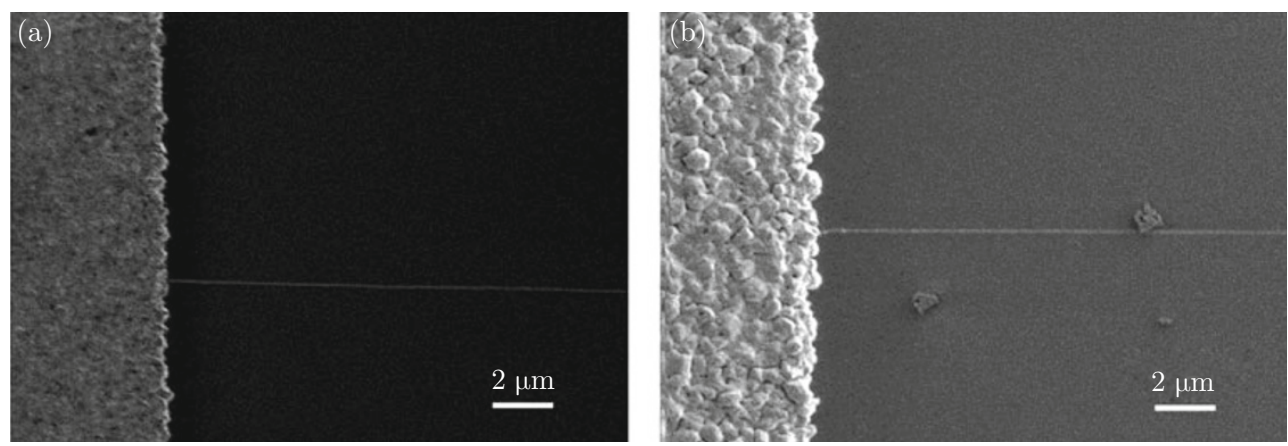

Fig. 4 SEM images of the fabricated gold (a) and copper (b) contacts. 
posited metal. As shown in Fig. 5(a), gold nanoparticles were observed on the side wall of SWNTs close to the electrodes when higher electroplating potential of $0.5 \mathrm{~V}$ was applied during gold electrodeposition. The amount of gold nanoparticles deposited on SWNTs increased when the depositing potential increased to 0.6 $\mathrm{V}$ (Fig. 5(b)), and gold covered the entire SWNT surfaces at the potential of $0.8 \mathrm{~V}$, forming gold nanowires along the SWNTs (Fig. 5(c), (d)).

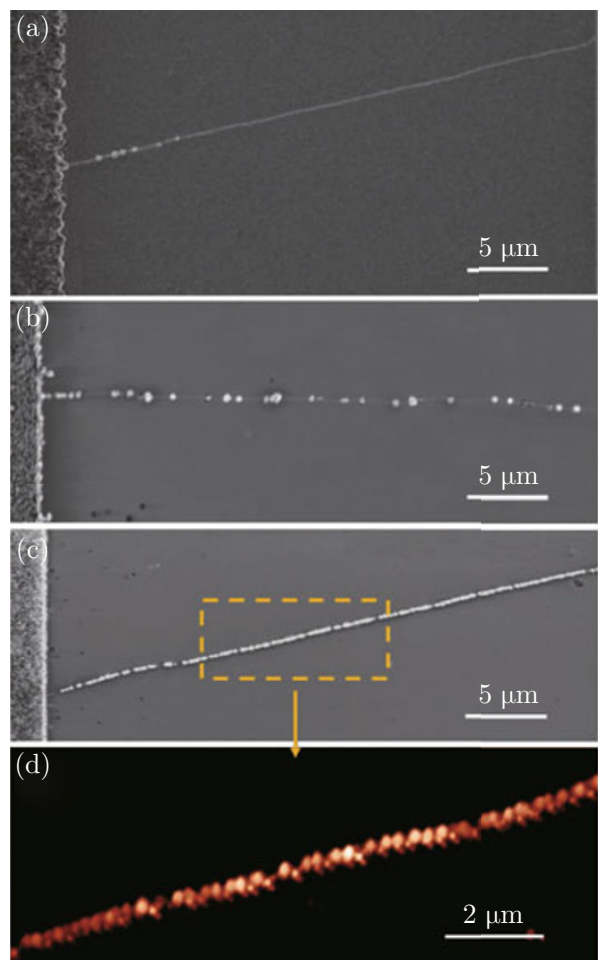

Fig. 5 SEM images of the SWNTs connected to Pt electrode after gold deposition at potentials of $0.5 \mathrm{~V}(\mathrm{a}) ; 0.6 \mathrm{~V}$ (b); and $0.8 \mathrm{~V}$ (c). (d) AFM image of the gold nanowire deposited on a SWNT in (c).

\section{Conclusion}

In summary, gold and copper contacts were fabricated on SWNT arrays by a maskless electrodeposition process. The contact fabrication method is applicable to both sSWNTs and mSWNTs, that is, a metal deposition region can be confined to the prepatterned electrodes, while leaving the section of all the SWNTs between the electrodes clean. Successfully deposited metal contacts under low potential can significantly decrease the SWNT-electrode contact resistance. The SWNT array remained a p-type semiconductor after electrodeposition. Gold contacts with a finer microstructure show better contact property than copper contacts. We believe, based on its parallel nature, our method has the potential in large-scale production of SWNT devices.

\section{Acknowledgements}

This research was supported by Shenzhen Key Laboratory of LED Packaging (No: ZDSY20120619141243215).

\section{References}

[1] B. Lassagne, Y. Tarakanov, J. Kinaret, D. GarciaSanchez and A. Bachtold, "Coupling mechanics to charge transport in carbon nanotube mechanical resonators", Science 325(5944), 1107-1110 (2009). http://dx.doi.org/10.1126/science. 1174290

[2] P. Avouris, "Molecular electronics with carbon nanotubes", Acc. Chem. Res. 35(12), 1026-1034 (2002). http://dx.doi.org/10.1021/ar010152e

[3] Y. L. Zhao, L. B. Hu, G. Gruener and J. F. Stoddart, "A tunable photosensor", J. Am. Chem. Soc. 130(50), 16996-17003 (2008). http://dx.doi.org/10. 1021/ja805393b

[4] J. L. Zhu, G. W. Zhang, J. Q. Wei and J. L. Sun, "Negative and positive photoconductivity modulated by light wavelengths in carbon nanotube film", Appl. Phys. Lett. 101(12), 123117 (2012). http://dx.doi. org/10.1063/1.4754624

[5] A. Bachtold, M. Henny, C. Terrier, C. Strunk and C. Schonenberger, "Contacting carbon nanotubes selectively with low-ohmic contacts for four-probe electric measurements", Appl. Phys. Lett. 73(2), 274-276 (1998). http://dx.doi.org/10.1063/1.121778

[6] H. Dai, J. H. Hafner, A. G. Rinzler, D. T. Colbert and R. E. Smalley, "Nanotubes as nanoprobes in scanning probe microscopy", Nature 384(6605), 147-150 (1996). http://dx.doi.org/10.1038/384147a0

[7] Z. Zhang and S. Zhang, "All-around contact for carbon nanotube field-effect transistors made by ac dielectrophoresis", J. Vac. Sci. Technol. B 24(1), 131-135 (2006). http://dx.doi.org/10.1116/1.2150226

[8] C. Chen, L. Yan, E. S. Kong and Y. Zhang, "Ultrasonic nanowelding of carbon nanotubes to metal electrodes", Nanotechnology 17(9), 2192-2197 (2006). http://dx. doi.org/10.1088/0957-4484/17/9/019

[9] D. W. Austin, A. A. Puretzky, D. B. Geohegan, P. F. Britt, M. A. Guillorn and M. L. Simpson, "The electrodeposition of metal at metal/carbon nanotube junctions", Chem. Phys. Lett. 361(5-6), 525-529 (2002).http://dx.doi.org/ 10. 1016/S0009-2614(02) 00992-2

[10] B. M. Quinn, C. Dekker and S. G. Lemay, "Electrodeposition of noble metal nanoparticles on carbon nanotubes", J. Am. Chem. Soc. 127(17), 6146-6147 (2005). http://dx.doi.org/10.1021/ja0508828

[11] S. Sahoo, S. Husale, S. Karna, S. K. Nayak and P. M. Ajayan, "Controlled assembly of Ag nanoparticles and carbon nanotube hybrid structures for biosensing", J. Am. Chem. Soc. 133(11), 4005-4009 (2011). http:// dx.doi.org/10.1021/ja1093327

[12] M. C. Tsai, T. K. Yeh and C. H. Tsai, " An improved electrodeposition technique for preparing 
platinum and platinum-ruthenium nanoparticles on carbon nanotubes directly grown on carbon cloth for methanol oxidation", Electrochem. Commun. 8(9), 1445-1452 (2006). http://dx.doi.org/10.1016/ j.elecom. 2006.07.003

[13] M. Zhang, Z. Zhou, X. Yang, X. Ye and Z. L. Wang, "Fabrication of $\mathrm{ZnO}$ nanowire devices via selective electrodeposition", Electrochem. Solid State 11(9), D69-D70 (2008). http://dx.doi.org/10.1149/ 1.2943658

[14] M. Zhang, Z. Zhou, X. Yang and X. Ye, "Pinning of single-walled carbon nanotubes by selective electrodeposition", Electrochem. Commun. 10(10), 15591562 (2008). http://dx.doi.org/10.1016/j.elecom. 2008.08 .003

[15] L. X. Zheng, M. J. O'Connell, S. K. Doorn, X. Z. Liao,
Y. H. Zhao, E. A. Akhadov, M. A. Hoffbauer, B. J. Roop, Q. X. Jia, R. C. Dye, D. E. Peterson, S. M. Huang, J. Liu and Y. T. Zhu, "Ultralong single-wall carbon nanotubes", Nat. Mater. 3(10), 673-676 (2004). http://dx.doi.org/10.1038/nmat1216

[16] D. Feng and G. Jin, "Introduction to Condensed Matter Physics: Volume 1", Singapore: World Scientific (2005).

[17] R. M. Penner, "Mesoscopic metal particles and wires by electrodeposition", J. Phys. Chem. B 106(13), 33393353 (2002). http://dx.doi.org/10.1021/jp013219o

[18] Y. Yao, Q. Li, J. Zhang, R. Liu, L. Jiao, Y. Zhu and Z. Liu, "Temperature-mediated growth of single-walled carbon-nanotube intramolecular junctions", Nat. Mater. 6(4), 283-286 (2007). http://dx. doi.org/10.1038/nmat1865 